\title{
Relationships between arsenic encapsulation, speciation, and bioaccessibility in mine tailings from the Empire Gold Mine, CA
}

\author{
MICAH K CHAR AND CHRISTOPHER S KIM
}

Chapman University

Presenting Author: char@chapman.edu

Waste material left over from the processing and extraction of metals in mining areas can contain associated and elevated levels of toxic metal(loid)s including arsenic (As), which can be mobilized into surrounding communities and incidentally ingested or inhaled by residents or recreational visitors to these sites. Additionally, physical and chemical weathering processes can affect contaminant bioaccessibility in ways that may influence risk estimates for surrounding residents.

Mine-impacted waste materials from the Empire Gold Mine in Grass Valley, California were collected as bulk grab samples from twenty sites including mine tailings and waste rock, then separated via mechanical sieving into eleven distinct size fractions ranging from $\geq 2830 \mu \mathrm{m}$ to $\leq 20 \mu \mathrm{m}$. Portions of select size fractions were then crushed with a ring mill to $\leq 10 \mu \mathrm{m}$. Nitrogen absorption surface area analysis and initial elemental concentrations (including arsenic) were measured for unground and ground samples.

In vitro simulated gastric fluid extraction tests were performed on both unground and ground size fractions to determine the bioaccessibility and relative change in bioaccessibility of As. Arsenic speciation on select unground and ground size fractions was also conducted through linear combination fitting of EXAFS spectra collected from beamline 11-2 at the Stanford Synchrotron Radiation Lightsource (SSRL).

Arsenic bioaccessibility was highly variable and correlated with both particle size and the presence of soluble As species. Controlled crushing experiments and As speciation analysis demonstrate that this correlation primarily arises through the removal of soluble As phases from within larger particle sizes. These findings demonstrate extended and possibly increasing As bioaccessibility in mine wastes over time, corresponding to subsequent increases in long-term exposure risk through incidental ingestion following physical weathering of As-bearing mine wastes. 